\title{
RFID Tags on Cork Stoppers for Bottle Identification
}

\author{
R. Gonçalves* ${ }^{* \dagger}$, S. Rima ${ }^{\ddagger}$, R. Magueta ${ }^{\dagger}$, A. Collado ${ }^{\ddagger}$, P. Pinho*, N. B. Carvalho*† and A. Georgiadis ${ }^{\ddagger}$ \\ *Instituto de Telecomunicações, 3810-193 Aveiro, Portugal \\ ${ }^{\dagger}$ DETI, University of Aveiro, 3810-193 Aveiro, Portugal \\ $\ddagger$ Centre Tecnologic de Telecomunicacions de Catalunya, 08860 - Castelldefels, Spain \\ Email:rgoncalves@av.it.pt,rlm@ua.pt, ppinho@deetc.isel.pt, nbcarvalho@ua.pt, srima@cttc.es, \\ acollado@cttc.es, ageorgiadis@cttc.es
}

\begin{abstract}
This paper presents the design of two UHF RFID tags in cork substrate. The RFID tags are designed to be compact and conformal to fit within the size and shape of the bottle and barrel stoppers. In a first step, the selected cork material is characterized taking into account the anisotropic properties of cork substrates and after the characterization two RFID tags are designed and optimized to cover a certain read range when placed inside bottle and barrel corks.

Index Terms-UHF RFID tag, cork substrate, conformal antenna amplifiers.
\end{abstract}

\section{INTRODUCTION}

Radio frequency identification (RFID) is currently a major enabling technology. Its being extensively used in many different kinds of applications and is one of the most promising bets for the development of the Internet of Things concept [1]. It has been widely used for a number of applications from identification and localization to various types of monitoring and sensing [2].

The diversity of scenarios of application of the RFID technology arise many challenges for the tag design. More and more the requirements shift towards the creation of small and conformal tags that can be embedded into the targets in a seamless way. To tackle those challenges the use of different substrate materials, specially materials that can be commonly found on consumable products, have been used. Materials such as paper, plastic (PET) [2], plywood [3], to name a few, as well as the use of different fabrication techniques targeting large volume production such as inkjet printing [4], and consideration of low cost conductive materials such as paperclips [4].

In this particular case we looked into the possibility of creating RFID passive tags for wine bottle labeling and identification. The challenge for conformal and low profile antennas, has led to different antenna designs, such as a meander monopole placed inside a hollow plastic bottle closure [5], and several designs consisting of dipole antennas placed around the bottle plastic or glass neck [6], [7] or body [8].

The designs that are around the bottle body and neck are most probably affected by the presence of liquid inside the bottle, while the liquid influence on the stopper is not so severe. Therefore, we chose to design our tags to be integrated into the stopper.

In this work, UHF tag antennas are designed using cork as the substrate material and copper tape as the conductive strip. Cork material is finding increasing application as an insulator in various fields including space technology, due to characteristic properties such as light weight and low thermal conductivity [9] while its extensive application in wine and bottle industry are well known. Standard milling fabrication is used to transfer the antenna design on a low cost adhesive copper tape, which is then attached to the cork substrate, allowing for a fast and cost effective fabrication.

First, the electrical properties of two types of cork samples are characterized. A natural cork sample and a fabricated pressed agglomerated cork sample. Then, two prototypes of UHF RFID tags are designed and fabricated. The performance of the tags is evaluated demonstrating a range of 3 to $6 \mathrm{~m}$. The designed tags can be used in bottle or barrel tagging applications. These can be expanded to consider the incorporation of a temperature sensor as part of a passive antenna sensing application.

The paper is organized as follows. In the following section the characterization of the cork materials used for the tag development is described and discussed. The third section presents the tag antenna designs and the measurement results. Finally the main conclusions are drawn in section IV.

\section{CORK CHARACTERIZATION}

In order to perform an optimum design of the RFID tags, it is necessary to accurately characterize the selected cork material. In the case of the cork substrate its anisotropic nature has to be considered and consequently, the characterization has to be done along the two different cuts of the cork material (axial and radial). The procedure followed to characterize the cork substrate is similar to the one in [10], where the Sparameters of two microstrip lines of different length (Fig. 1) are measured, their propagation characteristics determined and finally the dielectric constant and loss tangent values are obtained from these measurements.

The selected microstrip lines lengths are $l_{1}=15 \mathrm{~mm}$ and $l_{2}=25 \mathrm{~mm}$. The lines are fabricated by etching the microstrip lines on a copper tape using a standard milling process. The lines are attached to the top of the substrate material. The bottom of the substrate is covered by a layer of copper tape. Two sets of $l_{1} / l_{2}$ test lines where prepared. One set using pieces of cork cut in the axial direction and the other set using pieces of cork cut in the radial direction. 


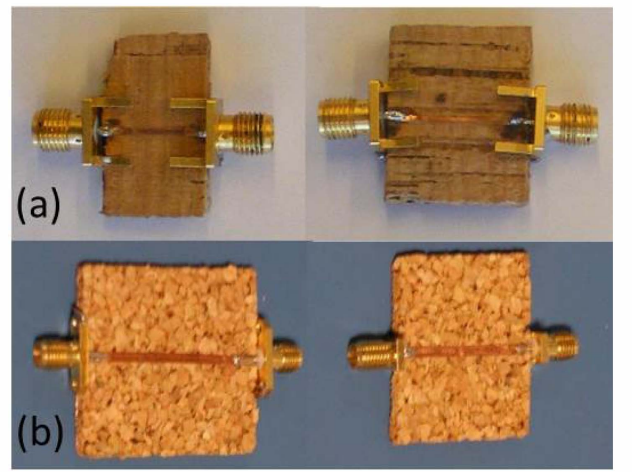

Fig. 1. Photograph of two microstrip lines placed on natural cork substrate: a) natural cork, axial cut and b) agglomerate cork, axial cut.

The S-parameters where measured in the 750 to $950 \mathrm{MHz}$ frequency range using the E8361A Agilent Vector Network Analyzer. Using the method in [10], both the dielectric constant and the loss tangent were obtained (Fig. 2 and Fig. $3)$. The dielectric constant is not very high as it could be expected from a material that has a lot of air gaps such as the cork. When comparing the characterization of the axial and radial samples, the anisotropic characteristics of the cork are confirmed, showing that the dielectric constant of the radial cork is around 1.6 and that of the axial cork around 1.9. In the same manner the loss tangent is slightly higher in the radial sample.

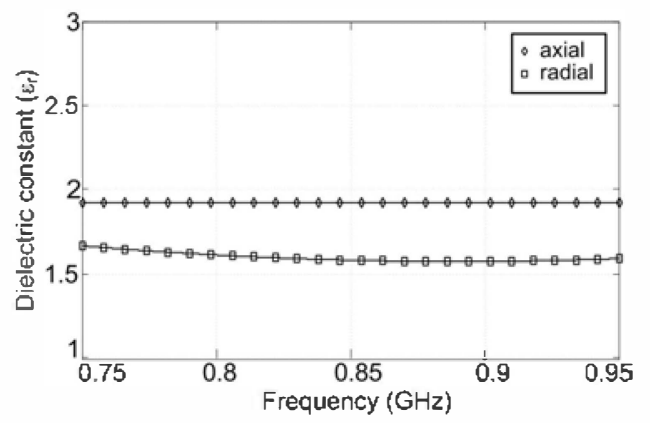

Fig. 2. Measured dielectric constant of axial and radial samples of natural cork in the range from 750 to $950 \mathrm{MHz}$.

For the agglomerate pressed cork the results were rather similar with a major difference being the even lower permittivity value measured, which was between 1.4 and 1.5 This was expected due to the higher number of air gaps in the material. Moreover, due to the random distribution of the cork pieces in the agglomerate cork laminate, the material is nearly isotropic.

\section{RFID TAG DESIGN}

Two different RFID tags were designed and tested. The first RFID tag is designed to fit inside barrel cork stoppers and the second RFID tag to fit into bottle cork stoppers. The tag designs are shown in Figures 4 and 5. The corresponding

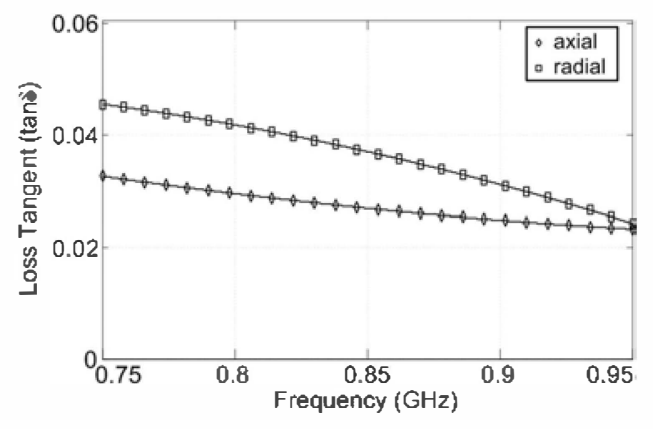

Fig. 3. Measured loss tangent of axial and radial samples of natural cork in the range from 750 to $950 \mathrm{MHz}$.

dimensions are detailed in Table I. The RFID tag for barrel stoppers uses a meandered dipole like antenna (Fig.4a) shaped to be placed in a cube shape cork material (Fig.4b). This cube is then placed inside the cork stopper (Fig.4c). The selected RFIC chip is a Higgs 3 from Alien Technology and presents an input impedance of $27.35-\mathrm{j} 200.7 \Omega$ at $866 \mathrm{MHz}$. The compact cube antenna is designed to have an input impedance that is conjugate match of that of the RFIC chip. The second RFID tag designed is intended to be placed inside a wine bottle cork stopper therefore the design is based on a meandered monopole antenna, so that the overall size is reduced (Fig.5). The used RFIC chip for this second tag is the UCODE SL3ICS1002 from NXP which has an input impedance of 16$\mathrm{j} 158 \Omega$ at $866 \mathrm{MHz}$.

The insertion of the stopper into the bottle applies some pressure into the cork, which might result in a deformation of the antenna and therefore lead to mismatches in the antenna. This is especially true for common cork stopper used in wine bottles, that are inserted under extreme pressure. In our case, we used the bottom of T-cork stoppers which are made of natural cork with a plastic top. These are commonly used in porto wine or brandy bottles. They can be inserted and removed several times from the bottle and when inserted they're under low pressure.

The bottle stopper is made of T-cork bottom which is inserted into the bottles and provides a secure grip while applying little deformation to the antenna. At the top, a bigger cork cylinder is used, staying outside the bottle. In this bigger part it is placed the chip and the matching inductive ring. This is clear in the antenna illustration presented in Fig. 5.

TABLE I

ANTENNA DIMENSIONS

\begin{tabular}{|l|c|}
\hline Parameters & sizes $(\mathrm{mm})$ \\
\hline \hline $\mathrm{L}_{\text {thickness }}, \mathrm{L}_{\text {meander }}$ & $0.75,14.0$ \\
\hline $\mathrm{L}_{\text {separation }}, \mathrm{W}_{\text {antenna }}$ & $1.0,25.0$ \\
\hline $\mathrm{L}_{\text {antenna }}, \mathrm{T}_{\text {antenna }}$ & $32.0,18.0$ \\
\hline $\mathrm{L}_{A}, \mathrm{~L}_{B}, \mathrm{~W}_{A}, \mathrm{~W}_{B}, \mathrm{~W}_{C}$ & $4.0,2.0,9.0,3.0,2.0$ \\
\hline $\mathrm{W}_{D}, \mathrm{~T}_{A}, \mathrm{~T}_{B}, \mathrm{~T}_{C}$ & $1.0,2.0,12.0,2.0$ \\
\hline $\mathrm{L}_{\text {separation_2}}, \mathrm{L}_{\text {thickness_2 }}$ & $0.65,1.15$ \\
\hline $\mathrm{L}_{1}, \mathrm{~L}_{2}, \mathrm{~L}_{3}, \mathrm{~L}_{4}, \mathrm{~L}_{5}, \mathrm{~L}_{6}$ & $26.0,30.0,40.0,3.0,4.5,7.4$ \\
\hline $\mathrm{L}_{7}, \mathrm{~L}_{8}, \mathrm{~L}_{9}, \mathrm{~L}_{10}, \mathrm{~L}_{11}$ & $17.0,23.0,10.0,3.0,3.5$ \\
\hline
\end{tabular}



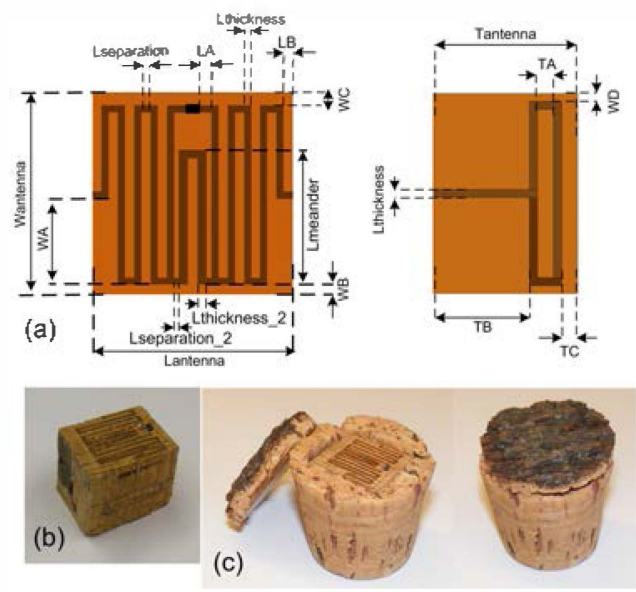

Fig. 4. Natural Cork RFID antenna (a) model (b) prototype and (c) inside barrel cork stopper.

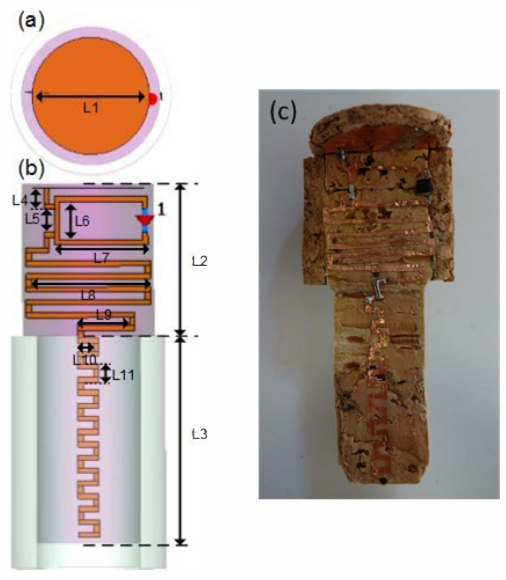

Fig. 5. Agglomerate Cork RFID antenna (a) top view (b) front view and (c) photograph.

In order to determine the maximum read range of the designed RFID tags, the setup of Figure 6 was used [11]. A signal generator is used to send the modulated carrier signal to the RFID tag, then the back-scattered signal is captured by an oscilloscope that allows determining if the tag is responding or not. The measurement procedure followed consist in placing the RFID tag at a certain fix distance from the transmitting antenna (in this case $\mathrm{d}=0.38 \mathrm{~m}$ ) and to evaluate the minimum power $\left(P_{\min }\right)$ level that is necessary to turn on the tag. Then the range can be calculated according to (1).

This measurement is repeated for each frequency. Then using (1) is possible to calculate the maximum read range at each frequency by relating the measured minimum frequency to the maximum transmitted power $P_{T}=30 \mathrm{dBm}$. The maximum read range for both of the two designed RFID tags are shown in Figure 7.

$$
r=d \cdot \sqrt{\frac{P_{T}}{P_{\min }}}
$$

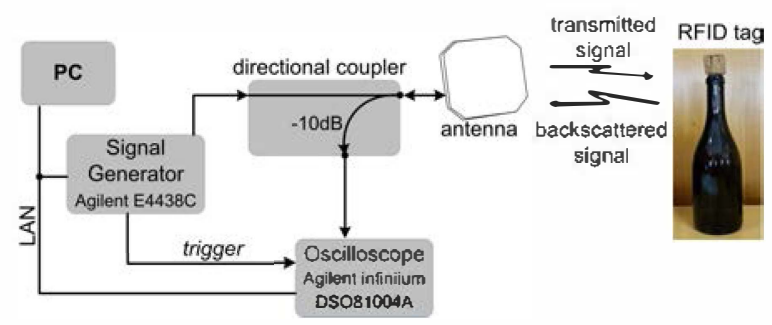

Fig. 6. RFID read range measurement setup.

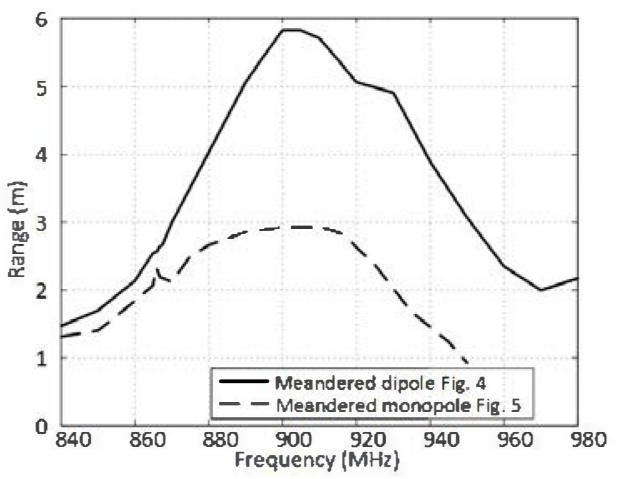

Fig. 7. Read range measurements for the cork RFID tags.

It is clear that the barrel stopper tag has a larger reading range. This happens given the antenna characteristics, since a dipole as slightly better gain than a monopole, which can lead to a noticeable reading range difference. Still, the other point to consider is that the barrel stopper was not tested when inserted into a liquid filled barrel. On the other hand, the bottle stopper was optimized to operate when inside a glass bottle and with liquid filling. The results showcased in Fig. 7 for the bottle stopper were measured with the tag inserted inside a water filled glass bottle.

Although the designed monopole was done in such way that it would present a radiation null in the direction of the liquid inside the bottle, given the large electromagnetic energy absorption from the liquids, the efficiency of the system drops slightly. That fact is the main source of loss in reading range, which is due to efficiency difference, between the barrel stopper and the bottle stopper results.

\section{CONCLUSION}

This paper presents the optimized design of two different UHF RFID tags using cork material as a substrate for the tag antenna design. The proposed RFID tags are intended to be integrated in bottle and barrel stoppers and provide information about the wine characteristics. In this paper the cork material is characterized showing the importance of considering its anisotropic nature when characterizing it. The implemented prototypes show good results in terms of read ranges which reach up to $6 \mathrm{~m}$. 


\section{ACKNOWLEDGMENT}

The authors would like to thank prof. Paula Seabra and Prof. Rob Pullar from CICECO-UA for their insight into the cork material properties and printing techniques. The authors acknowledge the Portuguese FCT/MCTES for financing the $\mathrm{PhD}$ grant SFRH/BD/91249/2012 and the project CREATION EXCL/EEI-TEL/0067/2012. This work was also supported by the Spanish Ministry of Economy and Competitiveness and FEDER funds through the project TEC2012-39143, and the EU Marie Curie FP7-PEOPLE-2009-IAPP 251557. Plus by EU COST Action IC1301 "Wireless Power Transmission for Sustainable Electronics (WIPE).

\section{REFERENCES}

[1] K. Finkenzeller, RFID Handbook: Fundamentals and Applications in Contactless Smart Cards and Identification, John Wiley \& Sons, Ltd, 2003.

[2] A. Rida., L. Yang, and M. Tentzeris, RFID-Enabled Sensor Design and Applications, Artech House, 2010.

[3] J. Virkki, J. Virtanen, L. Sydanheimo., L. Ukkonen, and M. M. Tentzeris, "Embedding inkjet-printed antennas into plywood structures for identification and sensing," 2012 IEEE International Conference on RFID Technologies and Applications, pp. 3439, November 2012.

[4] P. V. Nikitin, S. F. Lam, and K. V. S. Rao, "Rfid paperclip tags," 2011 IEEE International Conference on RFID Technologies and Applications, pp. 162169, April 2011.

[5] Z. Hu and P. H. Cole, "Bottle packaged wine product detection by uhf rfid systems," 2010 International Conference on Electromagnetics in Advanced Applications, pp. 301304, September 2010.

[6] Y. D. Kim, "Design of near omnidirectional uhf rfid tag with one-off seal function for liquid bottles," Wiley Microwave and Optical Technology Letters, vol. 55, pp. 375 379, 2013.

[7] J. Xi and T. T. Ye, "Conbottle uhf rid tag antenna mountable on winebottle neck," 2012 IEEE Antennas and Propagation Society International Symposium, July 2012.

[8] T. Bjorninen, A. Z. Elsherbeni, and L. Ukkonen, "Low profile conformal UHF RFID tag antenna for integration with water bottles," IEEE Antennas Wireless and Propagation Letters, vol. 10, pp. 1147 1150, 2011

[9] S. P. Silva, M. A. Sabino, E. M. Fernandes, V. M. Correlo, L. F. Boesel, and R. L. Reis, "Cork: properties, capabilities and applications," International Materials Reviews, Maney Publishing, vol. 50, pp. 345 365, 2005.

[10] F. Declercq, H. Rogier, and C. Hertleer, "Permittivity and loss tangent characterization for garment antennas based on a new matrix paencil two line method," IEEE Transactions on Antennas and Propagation, vol. 56, pp. $25482554,2008$.

[11] K. V. Seshagiri Rao, P. V. Nikitin, and S. F. Lam, "Antenna Design for UHF RFID Tags: A Review and a Pratical Application," IEEE Transactions on Antennas and Propagation, vol. 53, pp. 3870 - 3876, 2005 